\title{
ADVANCES IN CRYPTOLOGY
}

Proceedings of Crypto 83 


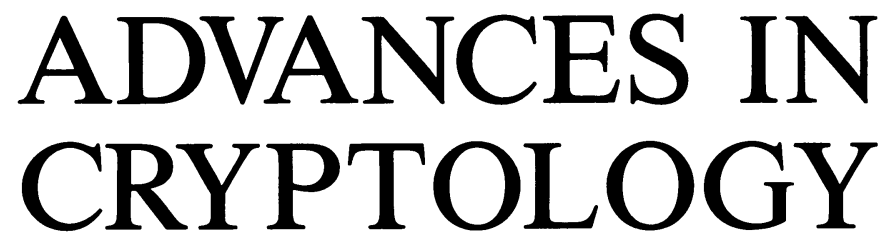

Proceedings of Crypto 83

Edited by

DAVID CHAUM

University of California

Santa Barbara, California

PLENUM PRESS • NEW YORK AND LONDON 
Crypto 83 (1983: University of California, Santa Barbara)

Advances in cryptology.

"Proceedings of a Workshop on the Theory and Application of Cryptographic Techniques, held August 21-24, 1983, at the University of California, Santa Barbara, California"-T.p. verso.

Includes bibliographical references and index.

1. Computers-Access control-Congresses. 2. Cryptography-Congresses. I. Chaum, David. II. Title.

QA76.9.A25W67 1983

001.64

84-3489

ISBN-13: 978-1-4684-4732-3 e-ISBN-13: 978-1-4684-4730-9

DOI: $10.1007 / 978-1-4684-4730-9$

Proceedings of a workshop on the Theory and Application of Cryptographic

Techniques, held August 21-24, 1983, at the University of California,

Santa Barbara, California

(C) 1984 Plenum Press, New York

Softcover reprint of the hardcover 1st edition 1984

A Division of Plenum Publishing Corporation

233 Spring Street, New York, N.Y. 10013

All rights reserved

No part of this book may be reproduced, stored in a retrieval system, or transmitted, in any form or by any means, electronic, mechanical, photocopying, microfilming, recording, or otherwise, without written permission from the Publisher 


\title{
CRYPTO 83
}

A Workshop on the Theory and Application of Cryptographic Techniques held at the University of California, Santa Barbara

August 22-24, 1983

Sponsored by

The International Association for Cryptologic Research

\section{Organizers}

\author{
Alan G. Konheim (UCSB), General Chairman \\ Neil J.A. Sloane (Bell Labs), Program Chairman \\ David Chaum (UCSB), Proceedings Editor \\ Paul Eggert (UCSB), Treasurer \\ Whitfield Diffie (BNR), Rump Session Chairman \\ Selim Akl (Queens University) \\ Henry Beker (Racal Research) \\ Tom Berson (SYTEK) \\ Thomas Beth (Universität Erlangen) \\ Dorothy Denning (SRI International) \\ Allen Gersho (UCSB) \\ John Gordon (Cybernation) \\ Robert Juneman (CSC) \\ Gus Simmons (Sandia Labs)
}




\section{Preface}

An international community of researchers is now flourishing in the area of cryptology-there was none half-a-dozen years ago. The intrinsic fascination of the field certainly is part of the explanation. Another factor may be that many sense the importance and potential consequences of this work, as we move into the information age. I believe that the various meetings devoted to cryptology over the past few years have contributed quite significantly to the formation of this community, by allowing those in the field to get to know each other and by providing for rapid exchange of ideas.

CRYPTO 83 was once again truly the cryptologic event of the year. Many of the most active participants continue to attend each year, and attendance continues to grow at a healthy rate. The informal and collegial atmosphere and the beach side setting which contribute to the popularity of the event were again supported by flawless weather. The absence of parallel sessions seemed to provide a welcome opportunity to keep abreast of developments in the various areas of activity.

Each session of the meeting organized by the program committee is represented by a section in the present volume. The papers were accepted by the program committee based on abstracts, and appear here without having been otherwise refereed. The last section contains papers presented at the informal rump session. A keyword index and an author index to the papers is provided at the end of the volume.

At CRYPTO 82 I proposed the formation of an International Association for Cryptologic Research to organize meetings and keep its members informed of events in the field. The association has taken the form of a non-profit corporation which held its first business meeting at CRYPTO 83. The attendees elected officers, a newsletter editor was selected, and plans were laid for EUROCRYPT 84 in Paris and CRYPTO 84 in Santa Barbara.

Many thanks are due the authors for their timely submission of papers, and to Ron Rivest and Alan Sherman for all their work in setting up the proceedings of CRYPTO 82.

D.C. January 1984 


\section{CONTENTS}

\section{SESSION I: ALGORITHMS AND THEORY}

Knapsack Public Key Cryptosystems and Diophantine Approximation (Extended Abstract) ................... 3

J.C. Lagarias

Solving Low Density Knapsacks ..................... 25 Ernest F. Brickell

Evaluation of the Adleman Attack on Multiply Iterated

Knapsack Cryptosystems (Abstract) .................. 39

E.F. Brickell, J.C. Largarias, and A.M. Odlyzko

On the Power of Cascade Ciphers (Extended Abstract) ........... 43

S. Even and O. Goldreich

The Prisoners' Problem and the Subliminal Channel............. 51

Gustavus J. Simmons

\section{SESSION II: MODES OF OPERATION}

An Overview of Factoring

H.C. Williams

New Ideas for Factoring Large Integers

Carl Pomerance, J.W. Smith, and S.S. Wagstaff, Jr.

Factoring Numbers on the Massively Parallel Computer

Marvin C. Wunderlich 
Factorization Using the Quadratic Sieve Algorithm. 103

J.A. Davis and D.B. Holdridge

\section{SESSION III: PROTOCOLS AND TRANSACTION SECURITY}

Signatures through Approximate Representations by

Quadratic Forms (Extended Abstract) ................ 117 H. Ong and C.P. Schnorr

A Simple Protocol for Signing Contracts .................. 133 Oded Goldreich

Reducibility among Protocols (Extended Abstract) .............. 137 Manuel Blum, Umesh V. Vazirani, and Vijay V. Vazirani

How to Exchange Half a Bit

Tom Tedrick

Blind Signature System (Abstract).

David Chaum

\section{SESSION IV: APPLICATIONS}

A Layered Broadcast Cryptographic System................ 157 M.E. Spencer and S.E. Tavares

Analytical Characteristics of the DES 171

Marc Davio, Yvo Desmedt, Marc Fosséprez, René Govaerts, Jan Hulsbosch, Patrik Neutjens,

Philippe Piret, Jean-Jacques Quisquater,

Joos Vandewalle, and Pascal Wouters

Producing a One-Way Hash Function from DES 203 Robert S. Winternitz

On the Security of Compressed Encodings ................. 209 Selim G. Akl 
Field Encryption and Authentication $\ldots \ldots \ldots \ldots \ldots \ldots \ldots \ldots \ldots \ldots \ldots \ldots$ Dorothy E. Denning

Shift-Register Synthesis (Modulo m) (Abstract)

J.A. Reeds and N.J.A. Sloane

\section{SESSION V: SPECIAL SESSION ON CRYPTANALYSIS}

Probabilistic Analysis and Performance Modelling of the 'Swedish' Algorithm and Modifications .............. 253

Joel E. Sachs and Shimshon Berkovits

A Subexponential-Time Algorithm for Computing Discrete

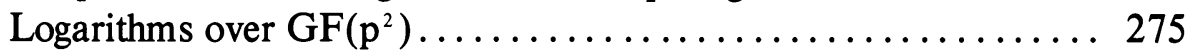

Taher ElGamal

Permutation Polynomials in RSA-Cryptosystems

Rudolf Lidl and Winfried B. Müller

Some Results on the Information Theoretic Analysis

of Cryptosystems

303

H. Jürgensen and D.E. Matthews

\section{RUMP SESSION: IMPROMPTU TALKS}

Improving the Security of Exponential Key Exchange........... 359 Bahaa W. Fam

RSA Bits Are 732 $+\epsilon$ Secure (Preliminary Abstract) ........... 369

Umesh V. Vazirani and Vijay V. Vazirani

Use of the 'Signature Token' to Create a Negotiable

Document ...

Donald W. Davies 
Electronic Wallet...

Shimon Even and Oded Goldreich

Design Concepts for Tamper Responding Systems............ 387 David Chaum

Author Index................................ 393

Subject Index............................... 395 